\title{
In vivo efficacy of tobramycin-loaded synthetic calcium phosphate beads in a rabbit model of staphylococcal osteomyelitis
}

\author{
Godday Anebow Lulu', Arunkumar Karunanidhi ${ }^{2,3}$, Loqman Mohamad Yusof ${ }^{4 *}$, Yusuf Abba ${ }^{5}$, Fazlin Mohd Fauzi ${ }^{3}$
} and Fauziah Othman ${ }^{1,6^{*}}$

\begin{abstract}
Background: Osteomyelitis is an acute or chronic inflammatory process of the bone following infection with pyogenic organisms like Staphylococcus aureus. Tobramycin (TOB) is a promising aminoglycoside antibiotic used to treat various bacterial infections, including $S$. aureus. The aim of this study was to investigate the efficacy of tobramycinloaded calcium phosphate beads (CPB) in a rabbit osteomyelitis model.

Methods: Tobramycin (30 mg/mL) was incorporated into CPB by dipping method and the efficacy of TOB-loaded CPB was studied in a rabbit osteomyelitis model. For juxtaposition, CPB with and without TOB were prepared. Twentyfive New Zealand white rabbits were grouped $(n=5)$ as sham (group 1), TOB-loaded CPB without S. aureus (group 2), S. aureus only (group 3), S. aureus + CPB (group 4), and S. aureus + TOB-loaded CPB (group 5). Groups infected with S. aureus followed by CPB implantation were immediately subjected to surgery at the mid-shaft of the tibia. After 28 days post-surgery, all rabbits were euthanized and the presence or absence of chronic osteomyelitis and the extent of architectural destruction of the bone were assessed by radiology, bacteriology and histological studies.

Results: Tobramycin-loaded CPB group potentially inhibited the growth of $S$. aureus causing 3.2 to $3.4 \log _{10}$ reductions in CFU/g of bone tissue compared to the controls. Untreated groups infected with S. aureus showed signs of chronic osteomyelitis with abundant bacterial growth and alterations in bone architecture. The sham group and TOBloaded CPB group showed no evidence of bacterial growth.

Conclusions: TOB-incorporated into CPB for local bone administration was proven to be more successful in increasing the efficacy of TOB in this rabbit osteomyelitis model and hence could represent a good alternative to other formulations used in the treatment of osteomyelitis.
\end{abstract}

Keywords: Tobramycin, Antibacterial activity, Calcium phosphate beads, Osteomyelitis

\section{Background}

Postoperative and post-traumatic osteomyelitis is one of the most serious complication with high rates of associated morbidity and has been categorized as

\footnotetext{
*Correspondence: logman@upm.edu.my; fauziah@upm.edu.my

${ }^{4}$ Department of Companion Animal Medicine and Surgery, Faculty

of Veterinary Medicine, Universiti Putra Malaysia, 43400 Serdang, Selangor Darul Ehsan, Malaysia

${ }^{6}$ Research Laboratory of Anatomy and Histology, Faculty of Medicine and Health Sciences, Universiti Putra Malaysia, 43400 Serdang, Selangor Darul Ehsan, Malaysia

Full list of author information is available at the end of the article
}

difficult-to-treat infections in the orthopaedic field [1, 2]. Tremendous progress has been made in the past few years aimed at preventing osteomyelitis, especially by using antibiotics with different spectrums of activity against specific pathogens. Operative techniques like debridement and muscle flaps have also been employed to overcome this complication [3-9]. In addition to the systemically administered antibiotics alone, the contemporary prevention strategies using biomaterials like antibiotic-loaded poly(methyl methacrylate) (PMMA) cement spacers and antibiotic-incorporated beads for local administration of antibiotics were comparably more 
effective in increasing the efficacy of locally-administered antibiotics [10-13].

Treatment of osteomyelitis with local administration of antibiotics using different delivery system, e.g. biodegradable microsphere containing TOB, PMMA beads containing TOB, in combination with systemic antibiotics are some of the choices of treatment $[13,14]$. The continued release of TOB from the microsphere and PMMA beads has been demonstrated in the treatment of osteomyelitis [15]. Microspheres containing TOB has also been reported to contain higher local antibiotic concentration, especially in bone and soft tissues compared to systemic levels [14, 15]. While the use of beads have shown considerable effectiveness in the treatment of osteomyelitis, disadvantages such as poor discharge of antibiotics, the inability to incorporate many useful antibiotics because of heat-instability, involvement of secondary surgery in the removal of cements and lack of biodegradable qualities makes them unviable treatment options [16-18]. Given that current treatment options for staphylococcal infections have become limited, this creates an urgent need for novel antibiotic delivery systems. Therefore, development of new delivery systems for antibiotics using biomaterials like CPB will not only remove $S$. aureus from infection sites effectively, but can potentially revert the damages inflicted on the bone by subsidizing mineral components, which is advantageous to new bone formation.

A biodegradable drug delivery system would have the merit of avoiding the removal of drug carrier which normally involves a secondary surgery. Furthermore, the discharge rate of antibiotics can be fine-tuned in a biodegradable system, which can be attributed to the properties of the material. The solubilizing property of the carrier does not prevent new bone from growing into the defective site, and the calcium component of the beads serves to replace the bone mineral.

Biodegradable materials like calcium sulfate, collagen, polymers and other microcapsules have been reported to be suitable drug delivery systems in the treatment of osteomyelitis [19]. In our earlier investigation, we have shown that in vitro incorporation of tobramycin and gentamycin with CPB resulted in a slow residual release of the antibiotic from $30 \mathrm{~min}$ to $1344 \mathrm{~h}$ (8 weeks) with dissolution of calcium phosphate [20]. However, incorporating TOB with CPB has not been tested in vivo in an animal model for the prevention of osteomyelitis. Therefore, the aim of the present study was to investigate the efficacy of TOB incorporated CPB in a rabbit osteomyelitis model.

\section{Materials and methods}

\section{Preparation of CPB loaded with TOB}

Commercially available calcium phosphate beads (JectOS $^{\circledR}$ liquid and powder, Kasios ${ }^{\circledR}$, L'Union, France) was prepared according to the methods described previously [20]. Briefly, the beads were prepared by pouring $5 \mathrm{~mL}$ of jectos liquid and $3 \mathrm{~mL}$ of $1 \%$ soluble starch (BDH, United Kingdom) into a sterile bowl. Then $10 \mathrm{~g}$ of jectos powder was added and mixed vigorously with spatula until the mixture turned to a smooth homogenous liquid ( $30 \mathrm{~min})$. Then, using a $1 \mathrm{~mL}$ syringe, the liquid paste was filled and slowly extruded on a petri dish to form $\sim 0.2 \mathrm{~g}$ bead. The prepared beads were allowed to dry overnight and sterilized by gamma irradiation (Malaysia Nuclear Agency) at a measured dose of $25.1 \mathrm{kGy}$. Tobramycin antibiotic (Sigma Aldrich, St. Louis, MO) was then loaded into $\mathrm{CPB}$ by dipping the beads into TOB solution at room temperature $\left(21^{\circ} \mathrm{C}\right)$ and allowing it to stand for $24 \mathrm{~h}$ at $4{ }^{\circ} \mathrm{C}$.

\section{Animals}

Twenty-five specific pathogen free adult male New Zealand White rabbits (Sapphire Enterprise, Selangor, Malaysia) weighing between 2.5 and $3 \mathrm{~kg}$ were used in this study. The animals were allowed to acclimatize for 3 weeks before surgery in the animal housing facility of Universiti Putra Malaysia (UPM), in a 12:12 light-dark cycle. All experiments were carried out in accordance with the guidelines of the Institutional Animal Welfare Committee; the study protocol was approved by the Animal Ethical Committee with approval number: UPM/ IACUC/AUP-R030/2013. Animals were housed in individual cages and were provided with antibiotic free rabbit diet (Perternakan Hong Lee Sdn Bhd, Malaysia) and tap water ad libitum. Rabbits were divided into five groups with four animals in each group and the details of grouping are as follows: group 1-normal control; group 2untreated group implanted with TOB-loaded CPB; group 3-untreated group inoculated with $S$. aureus; group 4treated group implanted with CPB and inoculated with $S$. aureus and group 5-treated group implanted with TOBloaded CPB and inoculated with S. aureus.

\section{Bacterial strain and inoculum preparation}

Reference strain of S. aureus ATCC 12600 (S. aureus strain Xen 29, ATCC Manassas, VA, USA) was used in this study [21]. The strain was grown in tryptic soy broth (TSB, Becton-Dickinson and Company) at $37^{\circ} \mathrm{C}$. Prior to surgery, bacterial inoculum containing $\sim 10^{7} \mathrm{CFU} / \mathrm{mL}$ in phosphate buffered saline (PBS) was prepared. Aliquot of $\sim 5 \times 10^{7} \mathrm{CFU} / \mathrm{mL}$ were obtained and re-suspended to a final volume concentration of $2 \times 10^{7} \mathrm{CFU} / \mathrm{mL}$. Cell 
suspensions were formulated on the day of surgery and held on ice until implantation. From this suspension, a volume of $100 \mu \mathrm{L}$ of inoculum was injected into the midshaft of the tibia. After surgery, the bacterial load present in $1 \mathrm{~g}$ of bone tissue was serially diluted and plated on TSA. After 28 days post-surgery, the presence of bacteria in bone tissues was evaluated based on the colony forming units (CFU) in $1 \mathrm{~g}$ of bone.

\section{Operative procedure}

Osteomyelitis of the tibia was induced by following the procedure described previously [22]. Briefly, the rabbits were weighed and anaesthetized with a combination of $5 \mathrm{mg} / \mathrm{kg}$ xylazine (Rompun, Bayer Vital GmbH, Leverkusen, Germany) and $35 \mathrm{mg} / \mathrm{kg}$ of ketamine (Ketaset, Zoetis, New Jersey, USA) via subcutaneous injection. Anaesthesia was maintained with free flow inhalation of $1.5 \%$ isoflurane (Primal Critical Care Inc., Pennsylvania, USA). Then the rabbits were placed on recumbence and the right knee and hock area was shaved and surgically prepared with chlorhexidine, 70\% alcohol and finally with tincture iodine. Prior to surgery, the surgical area was isolated from the rest of the body by placing a sterile surgical drape. A small skin incision of about 3-5 mm was made at the anterior surface of the tibia. The periosteum was sharply incised and bluntly elevated from the midshaft. Using an 18 guage needle, a hole of $\sim 0.2 \mathrm{~cm}$ was made at the midshaft of the tibia. Subsequently, the midshaft of 12 rabbits were inoculated with $100 \mu \mathrm{L}$ of $S$. aureus inoculum containing $2 \times 10^{7} \mathrm{CFU} / \mathrm{mL}$ by microinjection using a sterile pipette tip with an exterior diameter of $0.6 \mathrm{~mm}$ directly into the medullary canal. Immediately after challenging with $S$. aureus at the surgical site, a prepared CPB was inserted and placed directly over the hole at the midshaft of 12 rabbits (see Additional file 1: Fig. $\mathrm{S} 1)$. The fascial layer was then replaced to its initial position and closed immediately with a coated VICRYL ${ }^{\circledR} 4-0$ suture (Polyglactin 910, Ethicon Inc., New Jersey, USA) to prevent leakage of the inoculum and to secure the CPB in place. The control group $(n=4)$ received plain $\mathrm{CPB}$ without TOB. The TOB treated group $(n=4)$ received a total of $30 \mathrm{mg} / \mathrm{mL}$ of TOB. Osteomyelitis infection group $(n=4)$ were inoculated with S. aureus ATCC 12600 only. Test group $(n=4)$ were implanted with TOB-loaded CPB without bacteria. And the sham group $(n=4)$ was operated but left untreated. After surgery, the surgical sites were closed with stitches and disinfected, and all rabbits received $0.05 \mathrm{mg} / \mathrm{kg}$ of buprenorphine (PharmaForce Inc., Ohio, USA) as a post-operative analgesic, subcutaneously. Osteomyelitis of the right tibia was allowed to develop and was confirmed by X-ray after 28 days postsurgery. Animals were monitored daily for 28 consecutive days for locomotion status, food and water intake and signs of localized and systematic infection. Changes in body weight and body temperature were also recorded for their correlation to infection.

\section{Post mortem and sample acquisition}

On 28 days post infection (dpi), rabbits were euthanized with intravenous injection of $75 \mathrm{mg} / \mathrm{kg}$ pentobarbital sodium (Dolethal, Vetoquinol S.A., Lure cedex, France). The tibiae of each rabbit were subjected to radiographical, bacteriological, histological and macroscopic analysis. The infected bone and surrounding soft tissues of the tibia were removed. The right tibia was retrieved and stored in $10 \%$ buffered formal saline in airtight glass containers before it was processed for histological analysis. Prior to euthanasia, blood samples were drawn for hemogram analysis and erythrocyte sedimentation rate (ESR) evaluation at $0,7,14,21$ and $28 \mathrm{dpi}$. Bone samples were also obtained from experimental rabbits for bacteriological and histological analysis. The tibiae of the experimental rabbit was radiographed at three stages of the study i.e., before surgery, immediately after surgery and $28 \mathrm{dpi}$ in order to monitor the progression of osteomyelitis.

\section{Microbiological analysis}

Two transverse slices of $\pm 0.5 \mathrm{~cm}(\sim 0.9 \mathrm{~g})$ from the tibia were sawn; the proximal section was used for bacteriological determination, while the distal section was used for histological analysis. The proximal section was homogenized in $20 \mathrm{~mL}$ of PBS and the suspension was serially diluted in trypticase soy agar (TSA) plate. After $24 \mathrm{~h}$ incubation, the bacterial colony counts were enumerated to represent the CFU/g of bone. The average detection limit was set at $2500 \mathrm{CFU} / \mathrm{g}$ of bone and a fullblown infection was characterized by a bacterial load that reached $10 \%$ of the initial inoculum dose $\left(>10^{5} \mathrm{CFU} / \mathrm{g}\right)$. Colonies formed on TSA were further subcultured onto mannitol salt agar for the presumptive detection of $S$. aureus (Oxoid, Malaysia).

\section{Radiological analysis}

Odekerken's scoring system was used to confirm the signs of osteomyelitis, where a maximum score of four signifies severe osteomyelitis [23]. The specific morphological changes around the infected midshaft of the tibia were graded as follows: 0-no radiologic abnormalities; 1 -mild periosteal reaction with mild osteolysis; 2 - periosteal reaction and evident osteolysis; 3 -periosteal reaction with calcification in the subperiostium, extension of osteolysis to diaphysis and cortical thickening; 4-extensive metaphyseal osteolysis. 
Table 1 Osteomyelitis scoring system-histology (Masson Goldner's trichrome stain)

\begin{tabular}{ll}
\hline Osteomyelitis grade & Morphological changes \\
\hline 0 & No histopathological changes \\
1 & Mild periosteal inflammation \\
2 & Severe periosteal inflammation \\
3 & Acute intra-medullar inflammation \\
4 & Chronic intra-medullar inflammation \\
5 & Bone necrosis \\
\hline
\end{tabular}

Table 2 Microanatomy scoring system (Masson Goldner's trichrome stain)

\begin{tabular}{ll}
\hline Microanatomy grade & Microanatomy changes \\
\hline 0 & Decrease bone formation \\
& Destruction of bone architecture \\
& Increase bone formation \\
& Retention of bone architecture
\end{tabular}

\section{Histopathological analysis}

Bone samples were harvested and fixed in $10 \%$ buffered formal saline and histologically processed according to a previously described method with minor modifications [24]. Tissue sections were stained with Masson Goldner's Trichrome and the slides were examined under a light microscope (Olympus, Germany). The disease status was assessed based on the scores of acute intra-medullar inflammation, chronic intra-medullar inflammation, periosteal inflammation and bone necrosis (Table 1). New bone formation was assessed based on the microanatomy scoring system (Table 2).

\section{Statistical analysis}

Hematological data and the changes in body weight and temperature were analyzed by two way analysis of variance (ANOVA) followed by Tukey's multiple comparison of groups. The differences in bacterial loads between groups were determined by Mann-Whitney $U$-test for non-parametric two-tailed significance. The radiology, histology and microanatomy data were analyzed by Kruskal-Wallis test followed by Dunnett's or Tukey's multiple comparison test. A $p$ value of $<0.05$ was considered statistically significant and graphical representation of the data was performed using GraphPad Prism ${ }^{\circledR}$ version 6.01 (GraphPad, San Diego, CA, USA).

\section{Results}

Physical examination

All rabbits fully recovered after the surgery and had inappetence on the first day of surgery. There were no significant differences in the body weights of rabbits in all groups until at $28 \mathrm{dpi}$ (Fig. 1a). No profound rise in body temperature was observed in all groups, however significant differences in body temperature was observed in group infected with S. aureus ( $p \leq 0.001$; group 3 ) and group implanted with CPB and inoculated with $S$. aureus $(p \leq 0.01$; group 4$)$ on 7 and $14 \mathrm{dpi}$ as compared to $0 \mathrm{dpi}$ (Fig. 1b).

\section{Hematological analysis}

Postoperative hematological analysis showed no significant differences in total leucocyte counts in uninfected groups. However, significant differences in total leucocyte counts were found among the infection groups, i.e., group $3(p \leq 0.001)$, group $4(p \leq 0.001)$ and group $5(p \leq 0.05)$ on $7 \mathrm{dpi}$ as compared to $0 \mathrm{dpi}$. On 14, 21 and $28 \mathrm{dpi}$, groups 3 and $4(p \leq 0.001)$ showed increase in total leucocyte counts in comparison to baseline

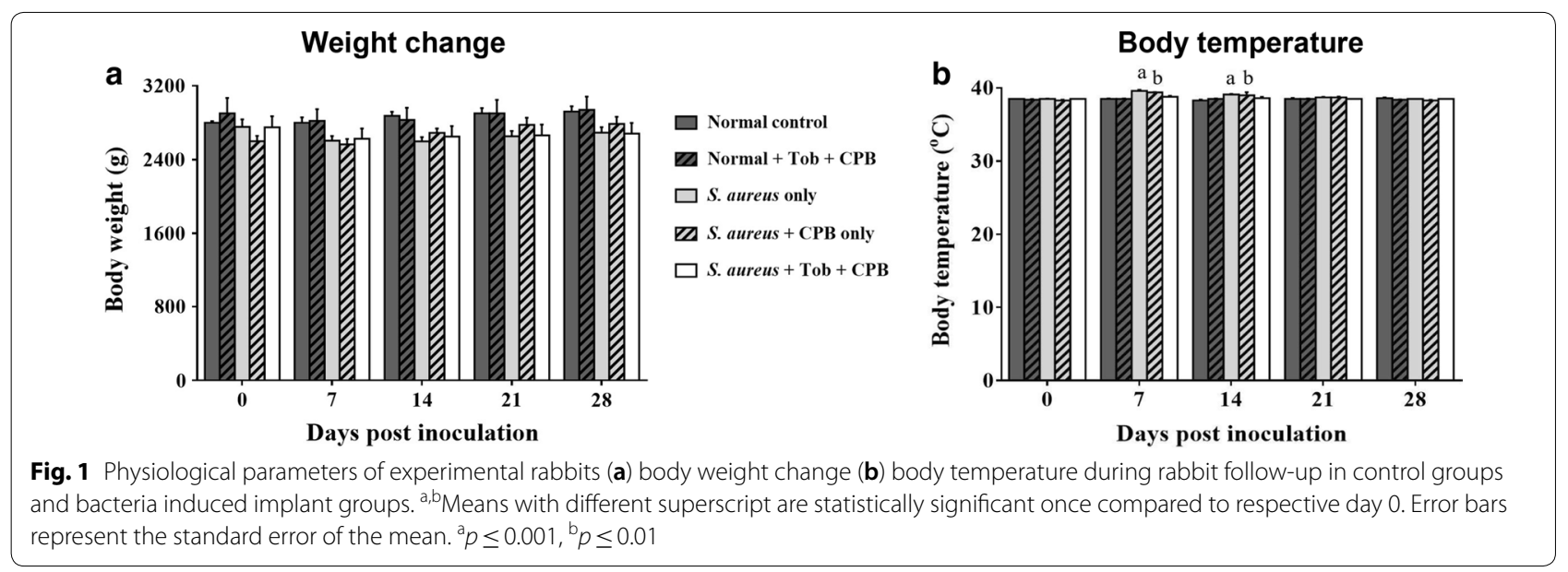


values ( $0 \mathrm{dpi})(p \leq 0.001)$. The total leucocyte count in the infected and uninfected groups were unaltered, the total leucocyte count remained unchanged in the uninfected groups as compared to the groups having contaminated implants with/without TOB (Fig. 2a). However, based on the trend, a steady decline in the

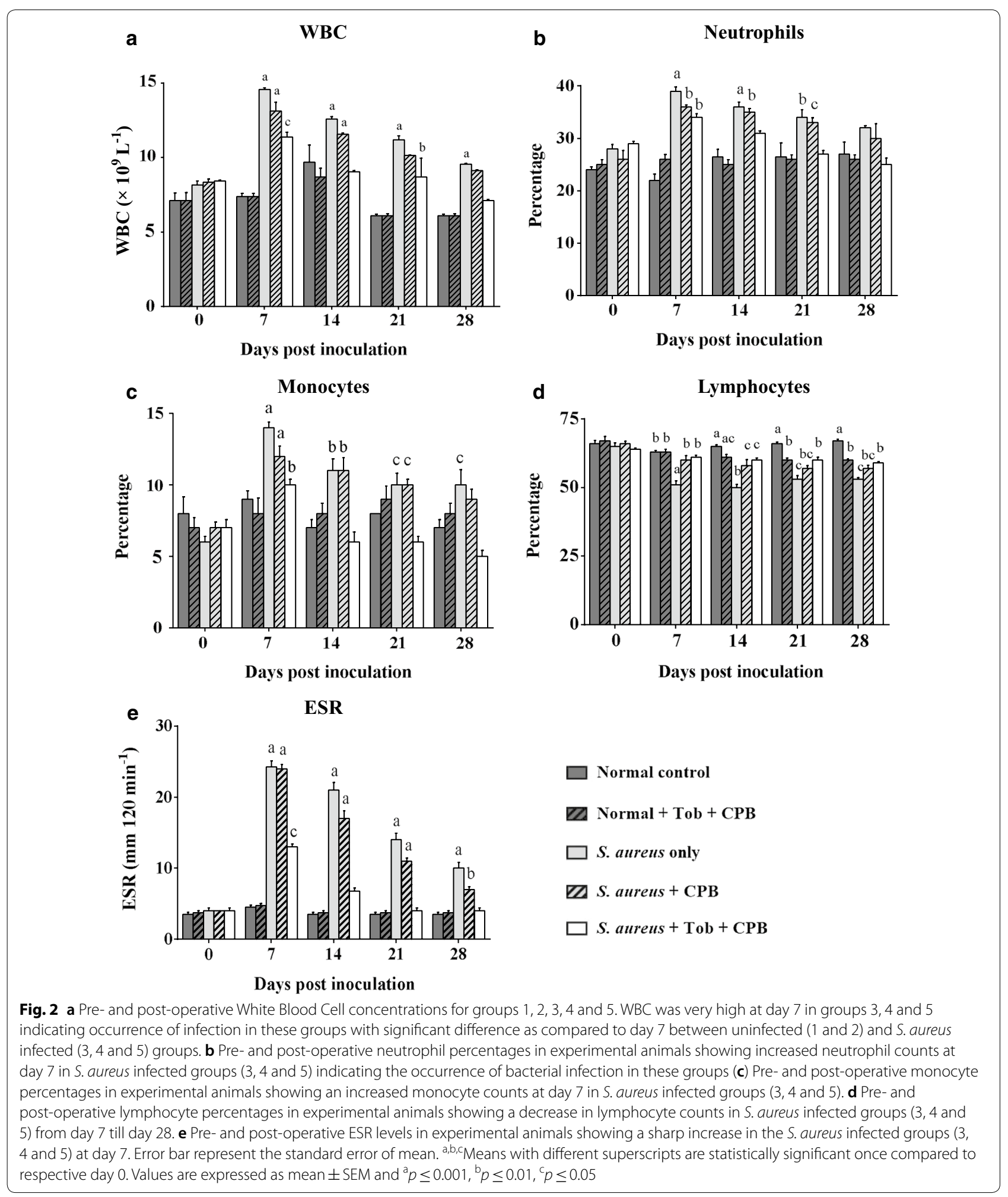


WBC count was observed in the infected groups from 7 to $28 \mathrm{dpi}$. The neutrophil counts in the infected groups (groups 3, 4 and 5) were increased at $7 \mathrm{dpi}$ in comparison to the baseline values ( $0 \mathrm{dpi})$. Similarly, at 14 and 21 dpi, groups 3 and 4 still had higher neutrophil counts in comparison to 0 dpi (Fig. 2b). The monocyte counts were increased $(p<0.05)$ in groups 3,4 and 5 at $7 \mathrm{dpi}$. At 14, 21 and $28 \mathrm{dpi}$, the monocyte count was higher $(p<0.05)$ in groups 3 and 4 . However, a decline trend was observed from $7 \mathrm{dpi}$ to $28 \mathrm{dpi}$, indicating gradual restoration of the levels to normalcy (Fig. 2c). The lymphocyte count consistently decreased $(p<0.05)$ in group 3 from 7 to $28 \mathrm{dpi}$, in comparison to counts seen at day 0 (Fig. $2 \mathrm{~d}$ ). The ESR level increased $(p<0.05)$ by three- to fivefold in the infected groups at $7 \mathrm{dpi}$. At 14 dpi, the ESR level was still higher $(p<0.05)$ in groups 3 and 4, but declined in group 5 to a level that was still higher than $0 \mathrm{dpi}$. At days 21 and 28, the ESR levels in groups 3 and 4 were still higher $(p<0.05)$ than at $0 \mathrm{dpi}$ (Fig. 2e).

\section{Tobramycin-loaded CPB prevented experimental rabbits from developing chronic osteomyelitis}

Several radiological parameters corresponding to osteomyelitis infection, such as changes in bone morphology, periosteal elevation and periosteal thickening together with meta- and diaphyseal osteolysis were observed in $\mathrm{X}$-ray radiographs, which facilitated the use of a modified

\section{a Tibiae of rabbit groups showing bone regeneration after 28 days}

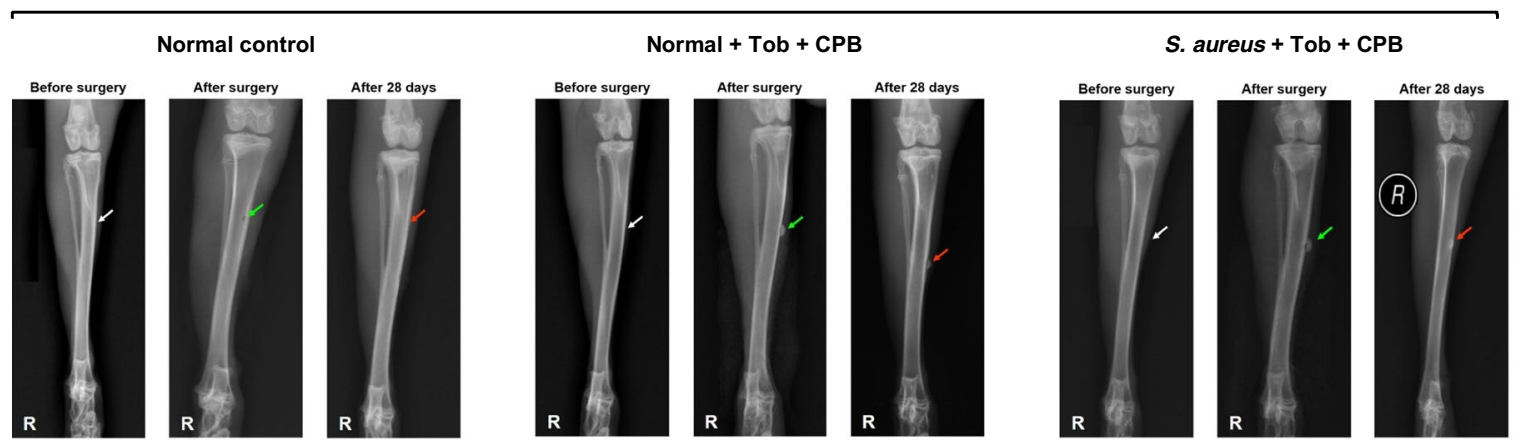

b Tibiae of rabbit groups showing bone destruction after 28 days

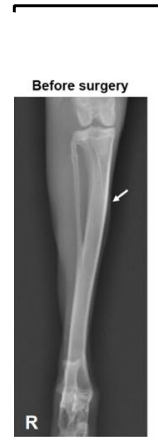

S. aureus only

S. aureus + CPB

C
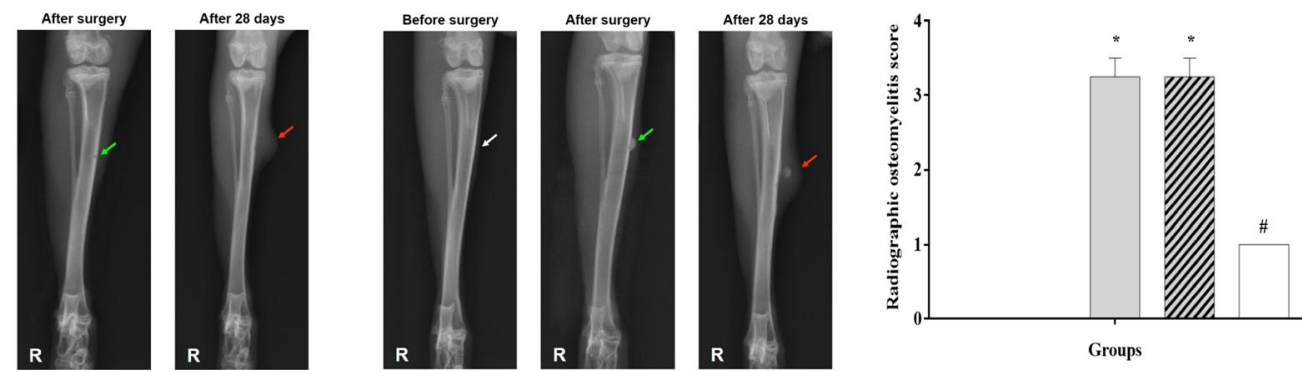

- Normal control

Z Normal + Tob + beac $\square$ S. aureus only ZZ S. aurens + bead only $\square$ S. aureus + Tob + bead

Fig. 3 a Radiological examination of Normal control, Normal + TOB + CPB; red arrow indicates evident bone formation, remodelling of the bone, dissolution of the CPB and filling of the bone defect with mineral component of the CPB 28 days postoperative. Radiological examination of S. aureus + $\mathrm{TOB}+\mathrm{CPB}$ (group 5); white arrow indicates area of the normal bone to be incised and radiological condition of the bone before incision; green arrow indicates incision point (bone defect) and implanted TOB incorporated CPB; red arrow indicates inhibition of inflammation by TOB, new bone formation, remodelling of bone, dissolution of CPB and filling of the bone defect with mineral component of CPB $28 \mathrm{dpi}$. $\mathbf{b}$ Radiological examination of S. aureus infected group (group 3); green arrow indicates point of incision (bone defect) and bacteria inoculation spot; red arrow indicates swelling due to inflammatory edema, abscess formation, soft tissue abnormality, inflammation, periosteal reaction, bone destruction, osteomalacia at 28 dpi (osteomyelitis formation). Radiological examination of S. aureus + CPB group (group 4); white arrow indicates area of the normal bone to be incised and radiological condition of the bone before incision; green arrow indicates point of incision (bone defect), point of bacterial inoculation and CPB implantation; red arrow indicates swelling due to inflammatory edema, abscess formation, soft tissue abnormality, displacement of the implanted CPB from the original position due to inflammation, periosteal reaction, bone destruction and osteomalacia at 28 dpi (osteomyelitis formation), $R=$ right tibia. c Quantification of X-ray images of experimental rabbits (groups 1-5) respectively. Error bars represent the standard error of mean. ${ }^{*} p<0.05$ 
scoring system [23] for osteomyelitis (Table 1). After 28 $\mathrm{dpi}$, the control groups showed no signs of abnormal morphology of the tibia; no closure of the bone defect and no displacement of the implanted CPB (Fig. 3a). However, the test group, i.e., S. aureus $+\mathrm{TOB}+\mathrm{CPB}$ showed mild or no periosteal reaction with closure of the defect at $28 \mathrm{dpi}$ (Fig. 3a). Whereas, signs of chronic osteomyelitis with periosteal reaction, osteolysis proximal to metaphyseal level and diaphyseal osteolysis with cortical resorption were noticed in the contaminated implant groups, i.e. S. aureus only and S. aureus $+\mathrm{CPB}$ at $28 \mathrm{dpi}$ (Fig. 3b). Assessments of the computed individual radiographs by use of the modified scoring system revealed a significantly greater score in the groups inoculated with S. aureus only and S. aureus + CPB (Fig. 3c).

\section{Tobramycin-loaded CPB exhibited strong antibacterial activity against $S$. aureus}

The quantitative bacteriological data from each group is shown in Fig. 4. Bone homogenate samples tested for the presence of $S$. aureus in the normal control and normal control + CPB groups showed the absence of $S$. aureus in these groups. Untreated groups, i.e. S. aureus only and $S$. aureus $+\mathrm{CPB}$ groups showed dense colonization of $S$. aureus growth ranging from $\sim 3.8$ to $4 \log _{10} \mathrm{CFU} / \mathrm{g}$ of bone tissue. A slight reduction in CFUs $\left(0.1 \log _{10}\right.$ reduction in CFU) was observed in S. aureus + CPB group compared to the control (S. aureus only). Similarly, the test group; S. aureus $+\mathrm{TOB}+\mathrm{CPB}$ showed 3.2-3.4 $\log _{10}$ reduction in $\mathrm{CFU}$ per gram of bone tissue compared to controls (Fig. 4). Microbiological analysis revealed that TOB-loaded CPB formulation exhibited strong anti staphylococcal activity.

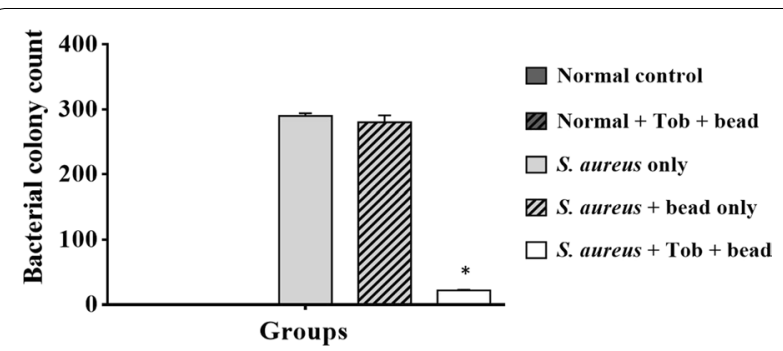

Fig. 4 Quantitative microbiological analysis. Average counts of CFU/g bone tissue ( \pm SEM) at day 28 are plotted. The $S$. aureus $+\mathrm{TOB}+\mathrm{CPB}$ group have significantly $\left({ }^{*} p=0.0014\right)$ reduced bacterial load compared with the control group 3 (S. aureus only). Group 4 (S. aureus + CPB) showed a slight tend towards reducing the bacterial load. Error bar represent the standard error of the mean
Tobramycin-loaded CPB groups exhibited enhanced new bone formation and remodeling of bone defects

Tissue sections from uninfected groups showed normal bone appearance, repaired bone defect and recanalization of medullary cavity (Fig. 5a). The treatment group, i.e. $S$. aureus $+\mathrm{TOB}+\mathrm{CPB}$ appeared normal with mild periosteal reaction, repaired bone defect and recanalization of the medullary cavity (Fig. 5a). However, the S. aureus only and $S$. aureus + CPB groups showed progressive destruction of the bone architecture and mineral component, without observable bone formation at the affected sites (Fig. 5b). The quantitative differences in osteomyelitis rating and microanatomy status between the various groups are shown in Tables 1 and 2, respectively. The scores indicated new bone formation in the uninfected controls (Normal control and $\mathrm{Normal}+\mathrm{TOB}+\mathrm{CPB}$ ) and the test group $(S$. aureus $+\mathrm{TOB}+\mathrm{CPB})$, respectively (Fig. 5c, d).

\section{Discussion}

In the present study, we used a prophylactic and a therapeutic model which was partially modified based on previously published models $[14,25]$. Moreover, many of the most relevant infection parameters were combined in a single animal model and these parameters were measured on a daily basis for 28 consecutive days. To additionally ensure a valid osteomyelitis model with a full blown infection in the $S$. aureus inoculated groups, the inoculum size was slightly increased. A $100 \mu \mathrm{L}$ inoculum of $S$. aureus containing $10^{6} \mathrm{CFU} / \mathrm{mL}$ is ideal to cause high rate of infection in the presence of carrier matrices like PMMA which is much similar to $\mathrm{CPB}[22,25]$. However, in this study $10^{7} \mathrm{CFU} / \mathrm{mL}$ was used which resulted in a full blown infection in the contaminated bead implant groups.

Antibiotic-impregnated beads, including CPB are commonly used to decrease the adverse systemic reactions and obtain local therapeutic levels of antibiotics [26]. In this study, TOB-incorporated $\mathrm{CPB}$, irrespective of the relatively large inoculum $\left(10^{7} \mathrm{cfu}\right)$, did not result in sepsis development in the animals. Another significance of this study is the excellent placement of the CPB in the tibial cavity. The placement of antibiotic beads has been shown to affect antibiotic tissue levels in the bone [15]. Localized treatment of osteomyelitis involves insertion of antibiotic beads into the tibial cavity thus adding to the clinical significance of this model [13].

With respect to the antibacterial potency of the TOBloaded $\mathrm{CPB}$, the results indicate that $30 \mathrm{mg} / \mathrm{mL}$ of TOB incorporated $\mathrm{CPB}$ was sufficient in inhibiting the growth of $S$. aureus in vivo, as evidenced by the lower bacterial load in comparison to the controls $(p=0.0014)$ (Fig. 4). The rabbit group inoculated with S. aureus only 


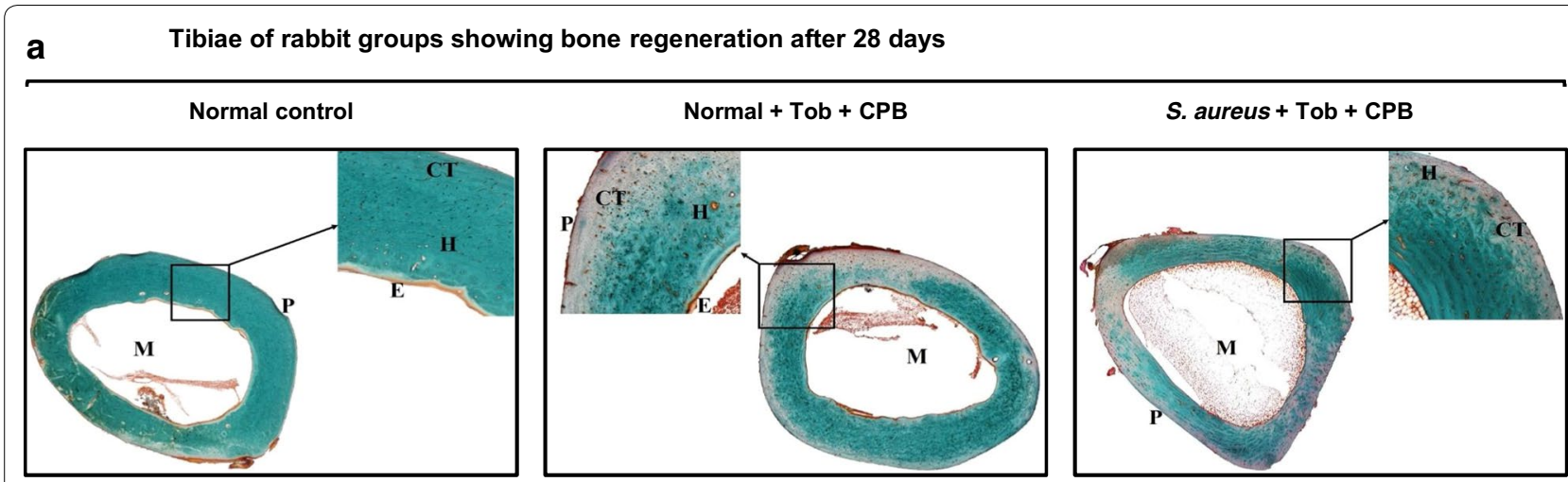

\section{b Tibiae of rabbit groups showing bone destruction after 28 days}

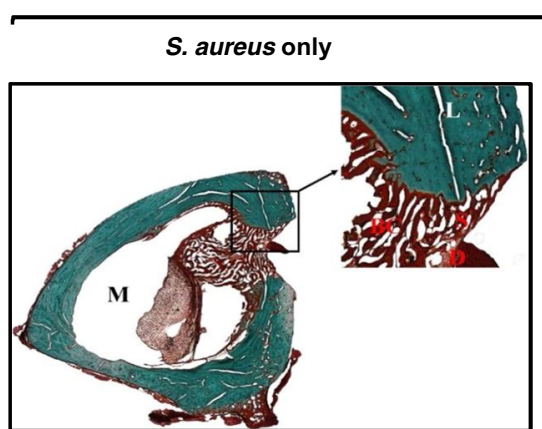

C

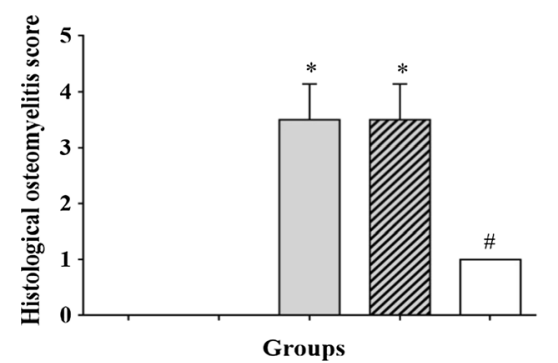

S. aureus $+\mathrm{CPB}$

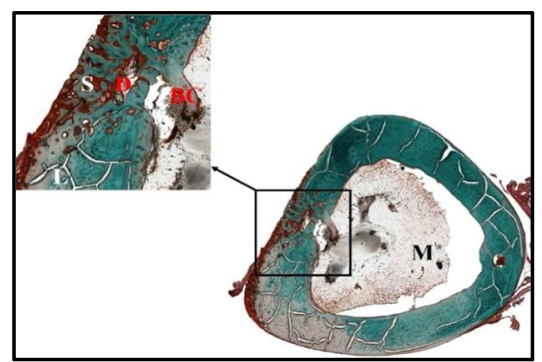

d

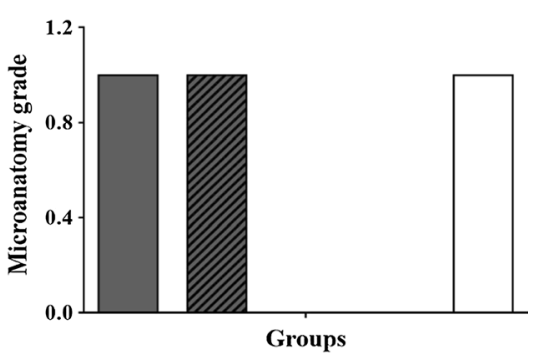

Fig. 5 Histological images of bone sections harvested at 28 dpi and stained with Masson Goldner's trichrome. a Group 1, group 2 and group 5 showing mild periosteal reaction consisting of normal bone architecture; slight distribution of immature connective tissue (CT), numerous active harversian canals $(\mathrm{H})$, endosteum-layer $(\mathrm{E})$ and marrow cavity. $\mathbf{b}$ Bone sections of groups 3 and group 4 showing osteomyelitis consisting of progressive destruction of bone architecture, mineral component of the bone and no bone formation at the defect site (D), note the bacterial colony (BC), empty lacunae (L) and scattered sequestra (S). ×40 magnification. c Quantification of histological images of experimental rabbits (groups 1-5) respectively, after 28 days follow up. Error bar represent the standard error of mean. ${ }^{*} p<0.05$. $\mathbf{d}$ Quantification of histological images of experimental rabbits (groups 1-5) after 28 days follow-up. Confirmation of new bone formation and microanatomical changes. $\times 40$ magnification

demonstrated a trend $(p \leq 0.001)$ towards increased bacterial growth per gram of bone. This in turn suggests a dose response effectiveness of TOB in vivo (Fig. 4). On the other hand, the group implanted with TOB incorporated $\mathrm{CPB}$ without bacterial inoculums showed no bacterial growth in their tibia (Figs. 4, 5a). Similar findings were observed in the sham group.

The dose of TOB incorporated in the CPB $(30 \mathrm{mg} / \mathrm{mL})$ was sufficient enough to prevent infection in the experimental rabbits. In our earlier investigation on the in vitro elution and dissolution studies using $\mathrm{TOB}$, incorporating TOB with $\mathrm{CPB}$ resulted in a slow residual release of the antibiotic from $30 \mathrm{~min}$ to $1344 \mathrm{~h}$ (8 weeks) and dissolution of calcium phosphate. Moreover, the mean TOB release in the TOB-incorporated $\mathrm{CPB}$ displayed a gradual increase from $10.0 \mu \mathrm{g} / \mathrm{mL}(168 \mathrm{~h})$ to $60.7 \mu \mathrm{g} / \mathrm{mL}$ (1344 h) which suggests that the rate of drug release was exponentially related to the release time [20]. This slow and steady release of TOB from $\mathrm{CPB}$ can be attributed to the bactericidal action of TOB against S. aureus as observed in 
this study. Furthermore, the dose of TOB released over time resulted in an amount that was optimal in killing the bacterial load present in $1 \mathrm{~g}$ of bone. It is therefore difficult to relate the very high release characteristics of TOB in vitro to the results obtained in vivo. Further confirmation is needed on the steady release and rapid clearance of TOB in the animal system. An earlier investigation by Den Hertog et al. [27] showed that TOB antibiotic is susceptible to degradation by proteolytic enzymes. This could possibly reduce local tissue levels of TOB in vivo [28]. In the present study, a consolidation of these factors could have resulted in diminished local complementary or active tissue concentrations of tobramycin, feasibly compromising the in vivo efficacy. This underlines the relatively better efficacy of TOB incorporated $\mathrm{CPB}$ in preventing osteomyelitis.

Previous studies have shown the importance of antibiotics incorporated to cement spacers or PMMA and their elution properties. One major advantage of these implants is that antibiotics are released from the carriers in such a way that the local levels of antibiotic vastly exceed the minimum inhibitory (MIC) or bactericidal (MBC) concentrations needed to treat most pathogenic organisms, and that these levels are much higher than those achieved with parenteral therapy [29]. Such high concentrations and a steady drug release are important, especially when TOB is used for impregnation of bone cement/CPB, because this aminoglycoside has a peak dose effect for bactericidal activity [30, 31]. A steady release and detectable concentration of TOB from carrier beads for 28 days has also been reported elsewhere [32].

It should be noted that a slight reduction in CFUs $(0.1$ $\log _{10}$ CFU reduction) was observed in the additional control i.e., group 2 (group receiving CPB with TOB). CPB is not known to possess any antibacterial activity; however, further investigation is required in order to confirm whether the slight reduction in CFUs observed in this group was exerted by $\mathrm{CPB}$ or due to immunological factors in the host. Besides, we only evaluated one bacterial species (S. aureus strain Xen 29), which means that the results obtained here may not be extrapolated in the scenario of osteomyelitis caused by clinical $S$. aureus in hospital settings. One limitation of this study is the use of mannitol salt agar only for the presumptive identification of $S$. aureus. The use of mass spectrometry MALDI-TOF for the confirmation of the presumptive $S$. aureus was not carried out in this study and would have confirmed the $S$. aureus identified. Furthermore, the use of an antibiotic resistant clinical isolate of $S$. aureus from a osteomyelitis positive patient would have had ample acerbity to steadily generate osteomyelitis in vivo which further broaden the applicability of this acute model. Thus, the data obtained here must therefore be considered only as indicative. We note that, similar in vitro observations have also been made for tobramycin alone and in combination with vancomycin [33-35], suggesting that detailed efficacy studies may be of interest.

Neutrophils are the first line of response in inflammation. In most bacterial infections, there is an accompanying leucocytosis with neutrophila [36]. Neutrophils recruited to the site of infection release chemotactic factors that attract other neutrophils and leucocytes to the site of injury. In this study, apparent leucocytosis with neutrophila was observed in all the infected groups at 7 $\mathrm{dpi}$, and subsequently in the infected untreated groups at 14 and $21 \mathrm{dpi}$. However, the group treated with TOB incorporated CPB had lower total WBC and neutrophil count in comparison to the infected untreated groups; this is due to the effect of TOB which resulted in reduced bacterial population at the site of injury and thus lower systemic leucocyte response.

\section{Conclusions}

The use of TOB-incorporated $\mathrm{CPB}$ at the sites of bone infection can be adopted as a standard care since $\mathrm{CPB}$ enabled the localization of supra-MIC levels which might have been difficult to achieve with other substitutes. Tobramycin-incorporated CPB effectively promoted soft tissue and bone healing, and prevented early bacterial colonization by $S$. aureus in infected tibia. CPB showed promising antistaphylococcal activity in treating chronic osteomyelitis when combined with TOB, with long periods of sustained efficacy. This profile is consistent with the properties required for the development of a potential antibacterial for staphylococcal osteomyelitis and supports continued investigation of this formulation. Future interests in the development of TOB as a traditional 'drug of choice' antibiotic will largely be focused on addressing issues related to its pharmacokinetics and inhibition of enzymatic proteolysis. Data obtained from the present study will serve as a basis for more progress in the development of TOB for the treatment of MDR pathogens in hospital settings.

\section{Additional file}

Additional file 1: Figure S1. Summary of surgical procedure to induce osteomyelitis and implantation of the CPB in the rabbits.

\section{Abbreviations}

ATCC: American Type Culture Collection; CFU: colony forming units; CPB: calcium phosphate beads; dpi: days postinfection; ESR: erythrocyte sedimentation rate; TSA: trypticase soy agar; MDR: multidrug resistant; PMMA: poly(methyl methacrylate); TOB: tobramycin; WBC: white blood cells. 


\section{Authors' contributions}

Conceived and designed the experiments: GAL, AK, LMY, FO. Performed the experiments: GAL, AK, LMY. Analyzed the data: GAL, AK, YA. Contributed reagents/materials/analysis tools: GAL, LMY, FO. Wrote the paper, and critically revised the manuscript for intellectual content: GAL, AK, YA, FMF. All authors read and approved the final manuscript.

\section{Author details}

${ }^{1}$ Department of Human Anatomy, Faculty of Medicine and Health Sciences, Universiti Putra Malaysia, 43400 Serdang, Selangor Darul Ehsan, Malaysia. 2 Department of Medical Microbiology and Parasitology, Faculty of Medicine and Health Sciences, Universiti Putra Malaysia, 43400 Serdang, Selangor Darul Ehsan, Malaysia. ${ }^{3}$ Department of Pharmacology and Chemistry, Faculty of Pharmacy, Universiti Teknologi MARA, 42300 Bandar Puncak Alam, Selangor Darul Ehsan, Malaysia. ${ }^{4}$ Department of Companion Animal Medicine and Surgery, Faculty of Veterinary Medicine, Universiti Putra Malaysia, 43400 Serdang, Selangor Darul Ehsan, Malaysia. ${ }^{5}$ Department of Veterinary Pathology and Microbiology, Faculty of Veterinary Medicine, Universiti Putra Malaysia, 43400 Serdang, Selangor Darul Ehsan, Malaysia. ${ }^{6}$ Research Laboratory of Anatomy and Histology, Faculty of Medicine and Health Sciences, Universiti Putra Malaysia, 43400 Serdang, Selangor Darul Ehsan, Malaysia.

\section{Acknowledgements}

The authors thank the staffs at Department of Orthopaedics, Traumatology and Rehabilitation, Faculty of Medicine, International Islamic University Malaysia for their assistance with this project.

\section{Competing interests}

The authors declare that they have no competing interests.

\section{Availability of data and materials}

The datasets used and/or analyzed during the current study are available from the corresponding author on reasonable request.

\section{Consent for publication}

Not applicable.

\section{Ethics approval and consent to participate}

This study was approved by the Animal Welfare Committee and the Animal Ethical Committee of Universiti Putra Malaysia (UPM/IACUC/AUP-R030/2013)

\section{Funding}

This work was supported Universiti Putra Malaysia through the Fundamental Research Grant Schemes (FRGS) 04-01-12-1131 FR funded by the Ministry of Higher Education (MOHE), Putrajaya, Malaysia.

\section{Publisher's Note}

Springer Nature remains neutral with regard to jurisdictional claims in published maps and institutional affiliations.

Received: 2 March 2018 Accepted: 12 December 2018 Published online: 28 December 2018

\section{References}

1. Al-Mudhaffar M, Prasad CV, Mofidi A. Wound complications following operative fixation of calcaneal fractures. Injury. 2000;31:461-4. https://doi. org/10.1016/S0020-1383(00)00026-7.

2. Ambrose CG, Gogola GR, Clyburn TA, Raymond AK, Peng AS, Mikos AG. Antibiotic microspheres: preliminary testing for potential treatment of osteomyelitis. Clin Orthop Relat Res. 2003;415:279-85. https://doi. org/10.1097/01.blo.0000093920.26658.ae.

3. Ferguson JY, Dudareva M, Riley ND, Stubbs D, Atkins BL, McNally MA. The use of a biodegradable antibiotic-loaded calcium sulphate carrier containing tobramycin for the treatment of chronic osteomyelitis: a series of 195 cases. Bone Jt J. 2014;96B:829-36.

4. Hauser CJ, Adams CA, Eachempati SR. Surgical infection society guideline: prophylactic antibiotic use in open fractures: an evidence-based guideline. Surg Infect (Larchmt). 2006;7:379-405. https://doi.org/10.1089/ sur.2006.7.379.

5. Holtom PD, Patzakis MJ. Newer methods of antimicrobial delivery for bone and joint infections. Instr Course Lect. 2003;52:745-9.

6. Sealy PI, Nguyen C, Tucci M, Benghuzzi H, Cleary JD. Delivery of antifungal agents using bioactive and nonbioactive bone cements. Ann Pharmacother. 2009;43:1606-15.

7. Srivastav A, Nadkarni B, Srivastav S, Mittal V, Agarwal S. Prophylactic use of antibiotic-loaded bone cement in primary total knee arthroplasty: justified or not? Indian J Orthop. 2009;43:259. https://doi.org/10.4103/00195413.53456.

8. Verron E, Khairoun I, Guicheux J, Bouler J-M. Calcium phosphate biomaterials as bone drug delivery systems: a review. Drug Discov Today. 2010;15:547-52. https://doi.org/10.1016/j.drudis.2010.05.003.

9. Zou Q, Li Y, Zhang L, Zuo Y, Li J, Li J. Antibiotic delivery system using nanohydroxyapatite/chitosan bone cement consisting of berberine. J Biomed Mater Res A. 2009;89:1108-17. https://doi.org/10.1002/jbm.a.32199.

10. Wentao Z, Lei G, Liu Y, Wang W, Song T, Fan J. Approach to osteomyelitis treatment with antibiotic loaded PMMA. Microb Pathog. 2017;102:42-4. https://doi.org/10.1016/j.micpath.2016.11.016

11. Kluin OS, van der Mei HC, Busscher HJ, Neut D. Biodegradable vs non-biodegradable antibiotic delivery devices in the treatment of osteomyelitis. Expert Opin Drug Deliv. 2013;10:341-51. https://doi.org/10.1517/17425 247.2013.751371.

12. Luo $S$, Jiang T, Yang $Y$, Yang $X$, Zhao J. Combination therapy with vancomycin-loaded calcium sulfate and vancomycin-loaded PMMA in the treatment of chronic osteomyelitis. BMC Musculoskelet Disord. 2016;17:502. https://doi.org/10.1186/s12891-016-1352-9.

13. Barth RE, Vogely HC, Hoepelman AIM, Peters EJG. "To bead or not to bead?"Treatment of osteomyelitis and prosthetic joint-associated infections with gentamicin bead chains. Int J Antimicrob Agents. 2011;38:371-5.

14. Ambrose CG, Clyburn TA, Louden K, Joseph J, Wright J, Gulati P, et al. Effective treatment of osteomyelitis with biodegradable microspheres in a rabbit model. Clin Orthop Relat Res. 2004;421:293-9. https://doi. org/10.1097/01.blo.0000126303.41711.a2.

15. Anagnostakos K, Wilmes P, Schmitt E, Kelm J. Elution of gentamicin and vancomycin from polymethylmethacrylate beads and hip spacers in vivo. Acta Orthop. 2009;80:193-7.

16. DiMaio FR. The science of bone cement: a historical review. Orthopedics. 2002;25:1399-407 (quiz 1408-9).

17. Calhoun JH, Mader JT. Antibiotic beads in the management of surgical infections. Am J Surg. 1989;157:443-9.

18. Klemm K. The use of antibiotic-containing bead chains in the treatment of chronic bone infections. Clin Microbiol Infect. 2001;7:28-31.

19. Diefenbeck M, Mückley T, Hofmann GO. Prophylaxis and treatment of implant-related infections by local application of antibiotics. Injury. 2006;37:2.

20. CheNorZarida CS, Fauziah O, Arifah AK, AzfarRizal A, Nazri MY, AhmadHafiz HZ, et al. In vitro elution and dissolution of tobramycin and gentamicin from calcium phosphate. African J Pharm Pharmacol. 2011;5:2283-91. https://doi.org/10.5897/ajpp11.591.

21. Funao H, Ishii K, Nagai S, Sasaki A, Hoshikawa T, Aizawa M, et al. Establishment of a real-time, quantitative, and reproducible mouse model of staphylococcus osteomyelitis using bioluminescence imaging. Infect Immun. 2012;80:733-41.

22. Nijhof MW, Stallmann HP, Vogely HC, Fleer A, Schouls LM, Dhert WJA, et al Prevention of infection with tobramycin-containing bone cement or systemic cefazolin in an animal model. J Biomed Mater Res. 2000;52:709-15.

23. Odekerken JCE, Arts JJC, Surtel DAM, Walenkamp GHIM, Welting TJM. A rabbit osteomyelitis model for the longitudinal assessment of early postoperative implant infections. J Orthop Surg Res. 2013;8:38.

24. Bhatt R, Lauder I, Finlay DB, Allen MJ, Belton IP. Correlation of bone scintigraphy and histological findings in medial tibial syndrome. Br J Sports Med. 2000;34:49-53.

25. Nijhof MW, Fleer A, Hardus K, Charles Vogely H, Schouls LM, Verbout AJ, et al. Tobramycin-containing bone cement and systemic cefazolin in a one-stage revision. Treatment of infection in a rabbit model. J Biomed Mater Res. 2001;58:747-53.

26. Calhoun JH, Mader JT. Treatment of osteomyelitis with a biodegradable antibiotic implant. Clin Ortho Related Res. 1997;341:206-14. 
27. Den Hertog AL, Wong Fong Sang HW, Kraayenhof R, Bolscher JGM, Vant Hof W, Veerman ECl, et al. Interactions of histatin 5 and histatin 5-derived peptides with liposome membranes: surface effects, translocation and permeabilization. Biochem J. 2004;379(3):665-72. https://doi. org/10.1042/bj20031785.

28. Wang Y, Johansson J, Agerberth B, Jörnvall H, Griffiths WJ. The antimicrobial peptide LL-37 binds to the human plasma protein apolipoprotein A-I. Rapid Commun Mass Spectrom. 2004;18:588-9. https://doi.org/10.1002/ rcm.1361.

29. Masri BA, Duncan CP, Beauchamp CP. Long-term elution of antibiotics from bone-cement: an in vivo study using the prosthesis of antibiotic-loaded acrylic cement (PROSTALAC) system. J Arthroplasty. 1998:13:331-8.

30. González Della Valle A, Bostrom M, Brause B, Harney C, Salvati EA. Effective bactericidal activity of tobramycin and vancomycin eluted from acrylic bone cement. Acta Orthop Scand. 2001;72:237-40. https://doi. org/10.1080/00016470152846547.

31. Boulanger S, Mitchell G, Bouarab K, Marsault É, Cantin A, Frost EH, et al. Bactericidal effect of tomatidine-tobramycin combination against methicillin-resistant staphylococcus aureus and Pseudomonas aeruginosa is enhanced by interspecific small-molecule interactions. Antimicrob Agents Chemother. 2015;59:7458-64. https://doi.org/10.1128/AAC.01711 $-15$.

32. Adams K, Couch L, Cierny G, Calhoun J, Mader JT. In vitro and in vivo evaluation of antibiotic diffusion from antibiotic-impregnated polymethylmethacrylate beads. Clin Orthop Relat Res. 1992;278:244-52.

33. Aiken SS, Cooper JJ, Florance H, Robinson MT, Michell S. Local release of antibiotics for surgical site infection management using high-purity calcium sulfate: an in vitro elution study. Surg Infect (Larchmt). 2015;16:5461. https://doi.org/10.1089/sur.2013.162.

34. Howlin RP, Brayford MJ, Webb JS, Cooper JJ, Aiken SS, Stoodley P. Antibiotic-loaded synthetic calcium sulfate beads for prevention of bacterial colonization and biofilm formation in periprosthetic infections. Antimicrob Agents Chemother. 2015;59:111-20.

35. Roberts R, McConoughey SJ, Calhoun JH. Size and composition of synthetic calcium sulfate beads influence dissolution and elution rates in vitro. J Biomed Mater Res Part B Appl Biomater. 2014;102:667-73.

36. Benson KG, Paul-Murphy J. Clinical pathology of the domestic rabbit: acquisition and interpretation of samples. El Paso: Veterinary Clinic of North America: Exotic Animal Practice; 1999. p. 539-51.
Ready to submit your research? Choose BMC and benefit from:

- fast, convenient online submission

- thorough peer review by experienced researchers in your field

- rapid publication on acceptance

- support for research data, including large and complex data types

- gold Open Access which fosters wider collaboration and increased citations

- maximum visibility for your research: over 100M website views per year

At BMC, research is always in progress.

Learn more biomedcentral.com/submissions 